
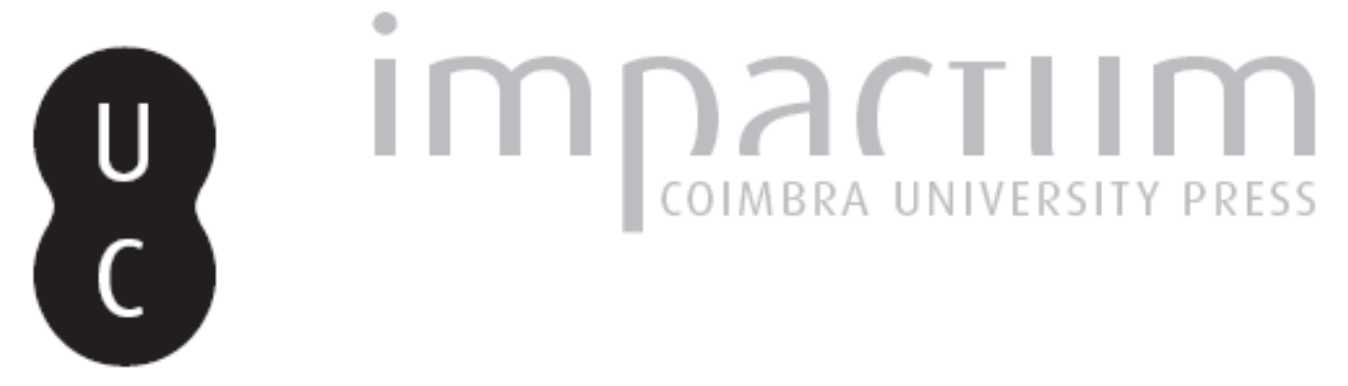

Qui a peur de l'éthologie? Action humaine et action animale chez Aristote

Autor(es): $\quad$ Morel, Pierre-Marie

Publicado por: Annablume Clássica

URL persistente:

URI:http://hdl.handle.net/10316.2/24520

DOI:

DOI:http://dx.doi.org/10.14195/1984-249X_11_8

Accessed : $\quad$ 26-Apr-2023 13:10:17

A navegação consulta e descarregamento dos títulos inseridos nas Bibliotecas Digitais UC Digitalis, UC Pombalina e UC Impactum, pressupõem a aceitação plena e sem reservas dos Termos e Condições de Uso destas Bibliotecas Digitais, disponíveis em https://digitalis.uc.pt/pt-pt/termos.

Conforme exposto nos referidos Termos e Condições de Uso, o descarregamento de títulos de acesso restrito requer uma licença válida de autorização devendo o utilizador aceder ao(s) documento(s) a partir de um endereço de IP da instituição detentora da supramencionada licença.

Ao utilizador é apenas permitido o descarregamento para uso pessoal, pelo que o emprego do(s) título(s) descarregado(s) para outro fim, designadamente comercial, carece de autorização do respetivo autor ou editor da obra.

Na medida em que todas as obras da UC Digitalis se encontram protegidas pelo Código do Direito de Autor e Direitos Conexos e demais legislação aplicável, toda a cópia, parcial ou total, deste documento, nos casos em que é legalmente admitida, deverá conter ou fazer-se acompanhar por este aviso. 

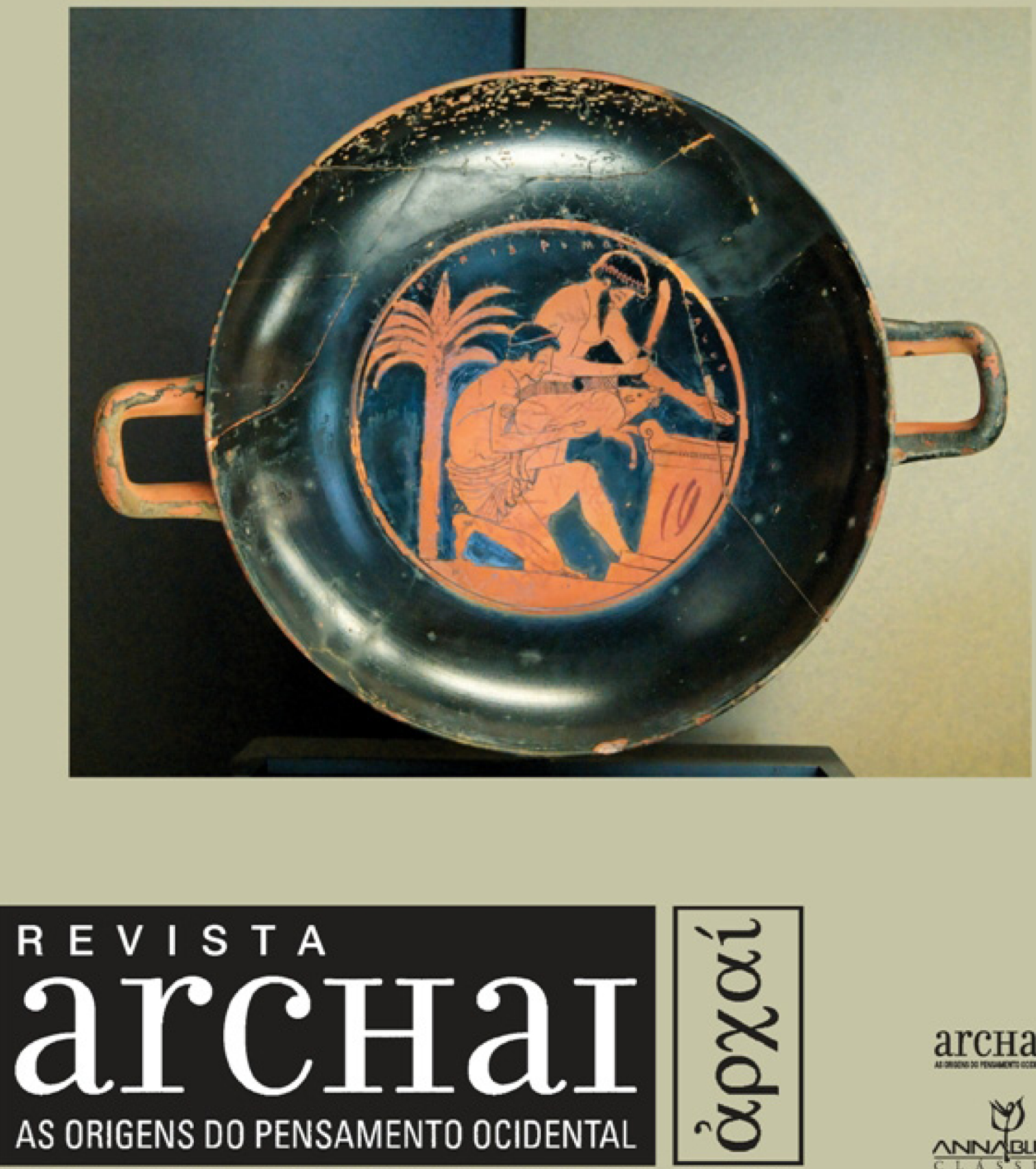

ARCHAI JOURNAL: ON THE ORIGINS OF WESTERN THOUGHT

arcHaI凰

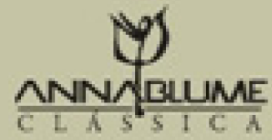




\section{QUI A PEUR DE L'ÉTHOLOGIE? ACTION HUMAINE ET ACTION ANIMALE CHEZ ARISTOTE}

MOREL, P.-M. (2013). Qui a peur de l'ethologie ? Action humaine et action animale chez aristote. Archai, n. 11, jul-dez, p. 91-100.

RÉSUMÉ: On décèle une tension entre deux tendances dans la philosophie aristotélicienne de la praxis : d'une part, une insistance claire sur la spécificité de l'action humaine par rapport à la conduite animale; d'autre part, la volonté de définir un genre commun pour les différents types d'activités, humaines et animales. Si cette tension peut être surmontée, c'est sans doute en distinguant les différents registres (axiologique, psychologique, éthologique) du discours aristotélicien. C'est aussi en précisant le type de ressemblance qui, à la fois, rapproche et distingue l'homme de l'animal : si la conduite humaine et la conduite animale appartiennent à un genre commun, elles demeurent distinguées par un rapport d'analogie, c'est-à-dire par une différence irréductible. L'analyse éthologique d'Aristote, loin de conduire à une naturalisation du comportement humain, conduit à sa spécification.

MOTS-CLÉS: Aristote, éthique, animal, action, éthologie, zoologie, analogie.

ABSTRACT: There is a tension between two features of Aristotle's conception of praxis: on the one hand, Aristotle emphasizes that human action differs specifically from animal behaviour; on the other hand, he seeks to define a common genus including the different kinds of activities (human and animal). In order to overcome this tension, one has to distinguish the axiological, psychological and ethological lines of Aristotle's arguments. One must also specify the sort of resemblance, which simultaneously differentiates and connects animals and human

Pierre-Marie Morel*

* ENS de Lyon - Institut Universitaire de France

Paris

France

1. Voir notamment Cicéron, De finibus, III, 16-19; $62-63$.

2. Voir la conception du juste exposée par l'épicurien Hermarque, rapportée par Porphyre, De abstinentia, I, 7-12.

3. Diogène Laërce, Vies, $X$ 137 ; Cicéron, De finibus, I, 71 ; II, 31-33. Sur cet argument commun aux épicuriens et aux stoïciens, on partira de l'étude fondamentale de Brunschwig (1986).

4. Pour Aristote, l'homme est un animal, de sorte qu'il faudrait préciser, chaque fois que l'on évoque la différence

anthropologique, que l'on oppose l'homme aux « autres » animaux. Par commodité cependant, je maintiendrai

ici l'usage moderne en disant « l'animal», « les animaux», là où en toute rigueur il faudrait dire « les autres animaux ».
Que l'éthique humaine ait quelque chose à voir avec l'animal ne va nullement de soi. L'idée paraîtra farfelue à certains, nécessaire à d'autres, s'il s'agit par exemple de défendre la cause des droits supposés de l'animal. Il reste que les grandes théories morales de la philosophie occidentale l'ont généralement écartée. Dans l'Antiquité, cette question a pourtant connu des développements considérables. Elle justifie toute la tradition du végétarisme, comme en témoigne le De abstinentia du néoplatonicien Porphyre. Elle explique également, pour partie, l'intérêt de la tradition stoïcienne pour le thème de l'appropriation (oikeiôsis) à soi et à la nature ${ }^{1}$, tendance dont le comportement animal fournit un exemple remarquable. Les épicuriens, bien qu'ils aient exclu les animaux de la sphère des relations contractuelles, et par conséquent de toute forme de communauté morale ou juridique ${ }^{2}$, ont vu dans le comportement animal - comme dans celui des nourrissons humains - un signe probant de notre tendance naturelle au plaisir ${ }^{3}$.

Si l'on choisit d'associer Aristote à cette réflexion, une première réaction vient d'emblée à l'esprit : la question ne se pose guère, en un sens, car les animaux ${ }^{4}$ ne comptent pour rien dans l'éthique telle que la conçoit Aristote. Dans nos actions, nous ne nous soucions guère des animaux, qu'il s'agisse 
beings. Although human and animal behaviour belong to the same genus, they relate to each other through analogy, that is to say through an irreducible difference. Thus, Aristotle's ethology, far from naturalizing human behaviour, leads to a better qualification of it.

KEY-WORDS: Aristotle, ethics, animal, action, ethology, zoology, analogy.

des égards ou des devoirs que nous pourrions avoir envers eux, ou bien qu'il s'agisse de discerner dans le comportement animal des exemples ou des modèles pour la conduite humaine.

La question de la prise en compte de l'animal peut cependant s'entendre en deux sens : l'existence des animaux entre-t-elle en jeu dans la mise en œuvre effective de la conduite morale ? L'existence des animaux entre-t-elle en jeu dans la théorie de la conduite morale, et jusqu'à quel point ?

Il est assez simple de répondre à la première question. La réponse ne peut être en effet que négative : je n'agis pas avec les animaux autrement qu'avec les objets inanimés, parce que nous ne formons pas avec eux une communauté éthique et parce que la valeur de mes actions ne dépend pas de la manière dont je me conduis avec eux. Le livre I de la Politique (I, 8, 1256b16-17) range très clairement les animaux parmi les éléments du domaine familial, en vertu de la chaîne téléologique qui veut que « les plantes existent pour les animaux et les animaux pour l'homme ». La seule relation naturelle entre l'homme et l'animal consiste donc dans le pouvoir que le premier exerce sur le second ${ }^{5}$. S'il est vrai que l'animal domestique y a quelque intérêt, car il trouve ainsi le moyen de sa préservation, ce n'est qu'une conséquence accidentelle du point de vue de l'usage humain. La question de la reconnaissance d'un droit des animaux, celle d'une compassion à l'endroit de l'animal, ou celle encore d'un bien-vivre animal se posent d'autant moins chez Aristote que le bien-vivre à proprement parler, ainsi que la moralité qui lui est coextensive, se définissent négativement par rapport à l'animal : le bonheur est une affaire proprement humaine, ce qui revient à dire qu'elle concerne, non pas l'animal en général, mais cet animal particulier qu'est l'homme. Ainsi, entre les animaux eux-mêmes, il ne saurait y avoir non plus de véritable communauté, pour cette raison précisément qu'ils s'assemblent en vue de vivre et non pas de bien vivre et ne " participent ni au bonheur, ni à la vie selon le choix » (Pol., I, 5, 1254b10-13).

Répondre à la seconde question est plus complexe. Elle est par ailleurs lourde d'implications, puisqu'elle engage le problème du naturalisme et de la différence anthropologique : si l'animal ne compte pour rien dans une éthique qui, de fait, s'affirme fortement comme proprement humaine, cela suggère que la conduite humaine, quoi qu'elle puisse devoir à une éventuelle «nature humaine » (expression étrangère à Aristote), ne relève pas - ou fort peu - des déterminations naturelles. Voyons cependant comment s'organisent ces différences entre l'homme et l'animal du point de vue de la question éthique. Considérons en effet que toute éthique présente trois aspects : un aspect axiologique, un aspect psychologique et un aspect éthologique (et éventuellement causal). J'entends par aspect axiologique l'ensemble des valeurs qui régissent l'éthique en question, comme le bien, le juste, ou dans un horizon plus large le sacré, l'amour de la patrie, etc. Par aspect psychologique, les facultés ou aptitudes (cognitives ou non) qui sont mobilisées par la conduite morale, ce qui nous amènera, dans le contexte de la philosophie d'Aristote, à nous demander si les animaux peuvent, en un sens au moins, avoir part à la conduite rationnelle, c'està-dire si leur comportement manifeste une faculté rationnelle de décision. Par aspect éthologique, je comprends (en donnant un sens large et non technique à ce terme) la structure du comportement, ou le mode opératoire mis en œuvre pour atteindre une fin donnée.

Or, si l'on peut assez rapidement, me semblet-il, s'accorder sur les deux premiers points, en montrant la spécificité et l'exclusivité de l'éthique humaine, le troisième mérite une attention particulière. Il invite en effet à s'interroger sur les modalités comportementales de la conduite, ce qu'Aristote désigne par un terme à la fois central et équivoque, celui de praxis. Il semble en effet que lorsqu'on analyse le comportement en termes de praxis, les frontières entre l'homme et l'animal se brouillent. Entre la nidification de l'hirondelle et 
l'acte de générosité en faveur d'un ami désargenté, la différence n'est plus si nette. Il y a en effet, on le verra, quelque chose de commun à l'activité animale et à l'activité humaine. Ainsi, l'approche éthologique peut donner l'impression que la différence anthropologique n'est pas radicale; en d'autres termes : que la différence entre conduite humaine et conduite

6. Sur cet aspect de la zoologie d'Aristote et sur les limites des rapprochements avec l'éthologie moderne, voir Labarriere (1993).

7. Pour une évaluation du «naturalisme » d'Aristote, je renvoie à Morel (2012)

8. Éthique à Nicomaque (EN), I 6, 1098a17

9. $E N, \mathrm{X}, 4,1174 \mathrm{~b} 31-33$

10. $E N, \mathrm{X}, 5,1176 \mathrm{a} 26-29$. 11. Voir : MOREL, 2007, p. 71-89.

animale $n^{\prime}$ est que graduelle ou relative ${ }^{6}$. $0 n$ voit bien

les enjeux sous-jacents : l'approche éthologique peut effrayer les lecteurs d'Aristote qui répugnent à lui attribuer une théorie causale de l'action humaine et qui veulent mettre cette dernière à l'abri de toute naturalisation ${ }^{7}$.

Pour le dire dès à présent, ce terme commun ne nous autorise pas à attribuer à l'animal un comportement assimilable à la conduite morale, ni à réduire l'action humaine à un épiphénomène comportemental. Il invite plutôt à s'interroger sur le type de ressemblance qui rapproche l'homme de l'animal, et à faire une différence d'analogie, par opposition à une différence spécifique ou à une différence simplement graduelle, entre la sphère de l'action humaine et la sphère de l'action animale. Or si ces sphères sont simplement analogues, et si les conduites par conséquent le sont aussi, ces dernières ne sauraient se confondre. L'analyse éthologique, on le verra, loin de conduire à la naturalisation, est en fait un instrument bien adapté pour montrer en quoi l'agir humain diffère du comportement animal.

\section{Spécificité axiologique et particularités psychologiques de l'activité humaine}

Il est tout à fait clair que, pour Aristote, l'homme a des fins spécifiques et que, s'il est vrai que le bien s'entend en plusieurs sens, le bien proprement humain réside dans une activité conforme à la plus haute vertu ${ }^{8}$, que cette dernière se réalise, ou bien dans une vie pratique - au sein d'une communauté d'action -, ou bien dans une vie contemplative, consacrée à l'exercice de l'intellect.

L'action humaine a donc un type de fin qui n'est pas réductible à une fin biologique : l'action vertueuse vise le bien et non le plaisir (que tout animal peut éprouver, et en vertu duquel il recher- chera ce qui est utile à sa survie), de sorte qu'elle doit se définir en termes de valeur ou de normes, et non pas seulement en termes de réplétion ou de compensation d'un manque. Si l'action procure du plaisir, c'est parce que celui-ci vient en surplus, comme l'achèvement de l'acte, sans être la fin même de l'action ${ }^{9}$. Aussi, bien que tous les plaisirs ne se vaillent pas, le plaisir n'a pas en tant que tel de valeur morale, parce que celle-ci dépend de l'action qui le produit. L'animal se meut en fonction d'une certaine représentation de l'utile et du nuisible, du plaisant et du douloureux, mais l'homme agit en vue d'un bien proprement humain. Du reste, seuls méritent d'être appelés « plaisirs propres à l'homme » ceux qu'éprouve l'homme moralement accompli $^{10}$.

Il n'y aura donc, en ce sens, qu'une relation d'analogie entre l'accomplissement d'une fonction naturelle ou biologique, et l'accomplissement pratique de cette fonction excellente qu'est la vertu. Ainsi, pour reprendre l'exemple d'Aristote en EN, I, 6 , la différence entre le cithariste et le bon cithariste n'est pas seulement de degré : le bon cithariste ajoute l'excellence à la fonction du cithariste (1098a10-11). L'œuvre qu'il réalise est dès lors tout autre : il réalise le bien de l'art du cithariste. Il remplit la fonction (jouer de la cithare), mais il ne s'en contente pas : il le fait bien, c'est-à-dire d'une manière qui excède le strict point de vue fonctionnel.

On pourrait objecter que le discours aristotélicien sur les opérations de la nature et sur les compétences de l'animal n'est pas dépourvu de connotations axiologiques. Ainsi, la sensation n'est pas seulement, pour l'animal, une fonction utile parmi d'autres : elle constitue son excellence propre. Elle est en effet la fonction ou « l'action » éminente, à laquelle toutes les autres sont subordonnées ${ }^{11}$. Quand il compare les animaux aux végétaux en Génération des animaux (GA), I, 23, Aristote rappelle que les premiers ont pour leur part une certaine faculté de connaissance (gnôsis), en l'espèce : la sensation. $0 r$ « elle varie beaucoup en

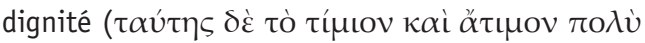

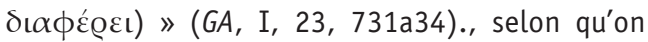
la compare à la faculté proprement humaine de connaissance ou qu'on la distingue des propriétés 
des êtres inanimés. Bien que la sensation, en effet, soit d'une dignité négligeable par rapport à l'exercice

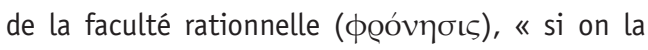
compare à l'absence de sensibilité, elle est ce qu'il y a de mieux ( (GA, I, 23, 731a34-731b2). Ainsi, lorsque l'animal se reproduit, il ne fait qu'accomplir la fonction qui est commune à tous les vivants, et « il devient comme une espèce de plante » (GA, I, 23, 731b5-8). Notons cependant que, même dans ce cas, l'animal, comme l'organe, se contente d'un accomplissement fonctionnel. S'il accomplit son « bien », c'est au sens où il réalise la fonction elle-même et non pas comme s'il s'agissait d'un surplus de valeur par rapport à celle-ci. L'excellence est dans la fonction même et non pas dans la manière dont elle est accomplie.

On ne peut enfin ignorer le discours fortement anthropocentriste qui est celui d'Aristote lorsqu'il caractérise l'action et la décision dans les traités éthiques. En affirmant que «la décision est le principe de l'action » (EN, VI, 2, 1139a31), Aristote invite à ne parler d'action que dans la sphère, proprement humaine, de l'éthique et de la politique. Il n'y a praxis, de ce point de vue, que lorsqu'il y a eu délibération et décision (ou choix : prohairesis), $c^{\prime}$ est-à-dire réflexion et désir ${ }^{12}$. L'action serait donc, en toute rigueur, spécifiquement humaine car « [...] la décision est ou bien un intellect désirant ou bien un désir raisonnant, et un tel principe est un homme »(EN, VI, 2, 1139b4-5). De même, selon l'Éthique à Eudème, l'homme est le seul vivant qui soit principe de certaines actions, si bien que « nous ne pourrions dire d'aucun autre vivant qu'il agit » (Éthique à Eudème (EE), II, 6, 1222b19-20).

Du point de vue psychologique, la différence est remarquable et elle porte précisément sur ce qui constitue en propre la conduite morale.

Aristote $a$, il est vrai, une conception très élaborée de la vie animale et des compétences mentales des animaux : l'homme lui-même est un animal ; l'animal autre que l'homme est en principe privé de la faculté rationnelle mais l'observation montre qu'il exerce une forme de discrimination cognitive qu'Aristote évoque parfois dans des termes qui servent également à désigner la faculté rationnelle proprement humaine : noûs, dianoia, sunesis ou phronêsis (un terme qui désigne chez Aristote non seulement la pensée en général, mais aussi la plus haute des vertus pratiques). Certains animaux communiquent entre eux au point qu'ils semblent apprendre les uns des autres ${ }^{13}$.

Pourtant, la distribution des facultés cognitives qui conduit à accorder une certaine raison aux animaux répond essentiellement, chez Aristote, à des préoccupations scientifiques et non pas à des préoccupations de type moral, comme l'a noté R. Sorabji au début du livre qu'il a consacré à la question $^{14}$. De ce point de vue, la thèse centrale d'Aristote est claire : seul l'homme possède la faculté rationnelle à proprement parler ; l'animal a seulement une sorte d'intelligence comportementale ${ }^{15}$. Je reviendrai sur la distinction qu'il convient de faire entre ces deux types de compétences rationnelles, ou plutôt sur ces deux registres du vocabulaire de la rationalité.

En un mot, aux yeux d'Aristote, ce qui nous lie à l'animal - si tant est que quelque chose nous lie effectivement à lui - n'est pas une communauté morale; nous n'avons pas de devoirs envers les animaux. Il serait vain de chercher dans le corpus aristotélicien une problématique claire et consistante du rapport éthique de l’homme à l'animal.

\section{La praxis, entre éthique et éthologie}

L'analyse des structures de la conduite résiste cependant à l'idée d'une rupture radicale entre action humaine et comportement animal. Il y a en effet une certaine homologie entre conduite animale et conduite humaine, au point qu'Aristote parle de praxis, activité, action, non seulement pour l'homme mais aussi pour l'animal. Il convient donc de revenir sur ce concept crucial, qui peut s'appliquer aux deux types de vies et qui nous installe au croisement d'un axe éthologique et d'un axe proprement éthique.

Il n'est pas naturel de parler aujourd'hui d'action pour qualifier un acte non-délibéré ou, plus généralement, le comportement animal. Nous concevons l'action comme une démarche et non comme un simple processus ou un pur acte. L'action nous apparaît comme une attitude consciente et volontaire, de sorte que ses conséquences nous sont
12. EN, III, 5, 1113a10-11.

13. Parties des animaux (PA), II, 17, 660a35-b3.

14. SORABJI, 1993, p. 2.

15. Voir en ce sens Sorabji (1993, p. 13) : « Aristotle himself points out that phronêsis is different in wild animals and in men. It is simply intelligence, or as he says, foresight ». Il renvoie à EN VI, 7, 1141a22-8. 
- au moins jusqu'à un certain point - imputables. Si l'on peut à la rigueur « ne pas être responsable de ses actes », ou ne pas être en tout point respon-

16. Voir en ce sens Pakaluk (2011), qui compare l'analyse aristotélicienne des actions mixtes à la notion contemporaine de «double effet » (comme tuer en situation de légitime défense, ou administrer un analgésique qui s'avère mortel, etc.) 17. Du Ciel, II, 12, 292a20-21. 18. Sur ce point et en faveur de la thèse selon laquelle ces emplois ne sauraient être de pures et simples métaphores, je renvoie à Morel (2007, p. 151-176) 19. PN, De sensu, 1, 436a4. Voir encore De Somno, 2, 455b28 : «I faut encore dire après cela par quels types de mouvements et d'actions (praxeis) internes aux corps se produisent la veille et le

sommeil chez les animaux.

20. HA, VIII, 1, 588a17. Voir aussi Pol., I, 4, 1254a7 : « la vie est action et non production

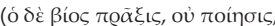
$\dot{\varepsilon} \sigma \tau \iota v)$

21. $P A, I, 5,645 \mathrm{~b} 29-33$. 22. $P N$, De sensu, 2, $438 \mathrm{a} 6 \mathrm{sq}$ mal que l'on puisse ne pas être responsable de ses actions immédiates.

Pourtant, Aristote fait un emploi très large de la notion de praxis. Le traité $D u$ ciel indique que les astres, comme les plantes, participent de la praxis et de la vie de la manière la plus simple : plus les êtres visent de biens différents, plus leurs " actions » sont diverses ${ }^{17}$. Le texte canonique sur la praxis, à savoir Métaphysique, $\Theta$, 6, 1048b18-36, la présente comme un processus complet, c'est-àdire un processus qui trouve sa fin en lui-même, inachevé parce qu'il est en marche progressive vers sa fin (comme la cure d'amaigrissement tend vers la minceur). On parlera donc de praxis, non seulement à propos des activités humaines, mais encore à propos de la vision ou du simple fait de vivre. Or sable des effets indirects de ses actes ${ }^{16}$, on conçoit par opposition au mouvement, qui est un processus ces activités sont à l'évidence communes à tous les animaux. Dans le corpus biologique, le terme praxis sert très fréquemment à désigner le fonctionnement d'un organe ou l'activité globale du vivant. Il est assez difficile de savoir ce qui unifie ces différentes occurrences de sorte qu'on a souvent considéré que l'usage de praxis dans un contexte autre qu'anthropologique était finalement métaphorique ${ }^{18}$. Toutefois, ces occurrences sont suffisamment nombreuses dans les PA ou l'Histoire des animaux (HA), et leur usage suffisamment rigoureux, pour que l'on puisse admettre qu'Aristote voit dans cette notion, non pas une simple manière de dire, mais un véritable concept opératoire. Les quelques exemples qui suivent devraient suffire à le montrer.

Les Parva naturalia entendent par exemple étudier des actions ou des activités (praxeis) ${ }^{19}$, conformément à cette affirmation plusieurs fois répétée dans le corpus biologique, selon laquelle l'accomplissement d'une fonction, celle de l'animal en son entier ou celle d'un simple organe, est une praxis.

Selon l'HA, dans un texte sur lequel nous reviendrons, les actions et les modes de vie des animaux diffèrent selon leurs mœurs et leur nour- riture $^{20}$. Activité vitale en tant qu'accomplissement de propriétés organiques, l'action animale est aussi bien une activité de l'organisme dans son rapport avec le milieu. Elle caractérise alors le comportement de l'animal. Le texte le plus clair sur ce point est sans doute celui de $P A, I, 5,645 b 14-20$, dans lequel l'action globale de l'animal est présentée comme la cause finale de l'ensemble des opérations organiques :

Puisque tout instrument est en vue de quelque chose, et que chaque partie du corps est en vue de quelque chose; que, d'autre part, ce qui est en vue

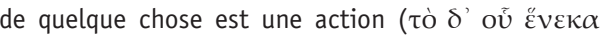
$\pi \mathrm{Q} \tilde{\xi}(\varsigma \tau \iota \varsigma)$; il est clair que le corps tout entier est constitué en vue d'une action complète. En effet, scier ne s'effectue pas en vue de la scie, mais c'est la scie qui est faite en vue de <l'action de> scier, car celle-ci est une sorte d'utilisation. Par conséquent, le corps lui aussi est en quelque sorte en vue de l'âme, et les parties sont également en vue des fonctions qui reviennent naturellement à chacune d'elles.

Ainsi, la respiration et les poumons sont fonctionnellement subordonnés au refroidissement de l'organisme. De même, la vue et l'œil sont fonctionnellement subordonnés à la connaissance. Ajoutons à cela les attributs nécessaires, le fait que l'existence de certains organes soit la conséquence nécessaire de celle d'autres organes ${ }^{21}$. Il est par exemple nécessaire que l'œil soit humide, et ainsi intérieurement diaphane, afin que passe la lumière grâce à laquelle s'opère la vision ${ }^{22}$. Nous sommes donc devant une série hiérarchisée d'activités, depuis la première activité des parties anhoméomères, jusqu'à l'activité totale du vivant. Un organisme se définit par une pluralité d'actions convergentes, par une sorte de cohérence pratique au sens propre.

Nous pouvons résumer la situation de la façon suivante. Chaque partie du corps existe en vue d'une fin, or la fin est une certaine action, donc l'ensemble du corps est constitué d'une action globale ou " complète », qui est elle-même le résultat d'une coalescence d'actions organiques, comme le montre la suite immédiate du texte de $P A$, I, 5. Les circonstances extérieures (la température, 
la présence d'air respirable, la nourriture, etc.) jouent assurément un rôle nécessaire, de sorte que l'organisme ne jouit jamais d'une autonomie absolue, mais il a une autonomie suffisante pour mener une vie qui lui est propre ${ }^{23}$. Par ailleurs, il n'est pas nécessaire que l'âme, entendue comme cause finale et principe unifiant de l'animal, soit présente de la même manière en toutes les parties. C'est ce que montre, dans le De Motu animalium (MA), l'image du monarque dans une cité bien organisée : de même que chaque citoyen accomplit bien la fonction qui est la sienne sans que le monarque ait à s'en occuper, de même chaque partie de l'animal remplit sa fonction sans qu'il y ait besoin d'une âme en chacune. Elles « vivent en union naturelle » (prospephukenai) avec le principe du corps, c'est-à-dire le cœur ${ }^{24}$. L'unité organique l'emporte ici sur le problème des mouvements dépendants des facteurs extérieurs : elle garantit assez l'autonomie de l'animal pour que nous puissions dire qu'il possède en lui-même le principe de son activité.

L'action organique et l'action animale obéissent donc à deux principes, que j'appellerai respectivement « principe de l'agent » et « principe de cohérence pratique ».

A) Principe de l'agent : l'organisation des « actions » et des mouvements qui les préparent permet de comprendre comment l'animal peut à la fois être mû et soumis à de multiples facteurs, tout en étant, dans son unité globale, l'agent de ses propres mouvements. De même, chaque organe, bien qu'il dépende des conditions organiques de son fonctionnement, se caractérise par une fonction immédiate (voir, respirer, se fléchir) qu'aucun autre organe ne peut accomplir à sa place. Toute action est donc réalisation, par l'agent même de l'action, d'un processus déterminé.

B) Principe de cohérence pratique : la vie du vivant est la synthèse ou la cohésion des activités qui assurent sa préservation. Elle définit donc la fin globale à laquelle ces activités se rapportent. Les actions, tout en ayant une fin propre (l'action, on l'a rappelé, est une activité dont la fin est immanente), et du fait même d'accomplir une fin propre, œuvrent également à un telos de niveau supérieur. Ainsi, le fléchissement des pattes, tout en étant la fonction immédiate et la fin du mouvement du membre, œuvre à la marche entendue comme processus global.

Faisons maintenant l'hypothèse que ce modèle est applicable, sous des conditions qu'il reviendra de préciser, à l'activité pratique. Il y a de fait une analogie incontestable entre la structure de l'action naturelle et celle de l'action humaine.

A) Le « principe de l'agent » est à l'œuvre par définition dans l'action délibérée, parce que la décision (prohairesis) dépend d'un principe immanent, la capacité de l'agent à déterminer sa conduite en vue d'une fin qu'il juge bonne. C'est pourquoi la décision est par définition « volontaire ». Aristote retient en effet, comme on le sait, deux critères de l'action volontaire, par opposition aux actes involontaires $^{25}$ : la connaissance par l'agent des circonstances qui entourent l'action et l'intériorité ou l'immanence du principe de l'action. La décision, qui est une sorte de désir, un désir intellectuel ou intellectualisé, est donc le signe de l'autonomie pratique, le fait même de l'agent moral. Bien que les textes que j'évoque ici soient riches d'implications et donnent lieu à une grande diversité d'interprétations, je me contente de les évoquer, pour ne retenir que ce qui est strictement nécessaire à la présente analyse : une action morale se caractérise d'abord par l'autonomie globale de son agent, qui peut ainsi être considéré comme responsable de l'action en question.

B) Le « principe de cohérence pratique » trouve lui aussi sa traduction dans la sphère de l'activité humaine : l'action humaine consiste le plus souvent en une organisation des fins particulières en vue d'une fin ultime. L'Éthique à Nicomaque, dans un texte fondamental pour la théorie aristotélicienne de l'action, montre que les activités, pratiques ou productives, ont une complexité interne qui a pour conséquence une démultiplication des fins : certaines actions servent à en réaliser d'autres, comme la technique du harnachement sert l'art hippique et celui-ci l'art de la guerre ${ }^{26}$. Les arts ou techniques, parce qu'ils ont pour fin ultime la production d'un objet ou d'un état extérieur à l'agent - comme la santé du patient dans l'art médical -, diffèrent il est vrai des activités pratiques, dont la fin ultime est immanente à l'agent. L'analogie entre les arts
23. Je renvoie sur ce point à Morel (2007, p. 137-147). 24. Voir MA, 10, 703a29-703b2. Sur ce texte, je renvoie à mes commentaires dans : MOREL, 2013. Voir aussi PA, III, 7, 670a25-26 : le cœur est un foyer qui est comme l'acropole du corps. 25. Voir EN, III, 1-3 et EE, II, 6-9. 26. EN, I, 1, 1094a10-13. 
et la pratique est néanmoins légitime, lorsqu'il s'agit de comprendre l'enchaînement des fins et leur unification sous le principe d'une fin ultime. En effet le prakton, le faisable ou la chose à accomplir dans le domaine de l'action comme dans celui de la technique, peut s'entendre en deux sens, dont l'un renvoie à la fin ultime et l'autre aux fins qui valent comme moyens au regard de la fin ultime :

Les choses en vue desquelles nous agissons et celles que nous faisons en vue d'elles participent à l'action

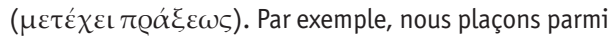
les choses à accomplir ( $\tau \tilde{\omega} \nu \pi \varrho \alpha \kappa \tau \tilde{\omega} \nu$ ) la santé et la richesse et aussi ce que nous faisons en vue d'elles, les activités saines et les activités rentables. (EE, I, 7, 1217a36-39).

Sous un certain aspect, l'action morale

27. EN, VI, 2, 1139b4 28. $H A, I, 1,488 \mathrm{~b} 12-28$ 29. $H A, I X, 47,630 b 31-631 a 7$ 30. Voir par exemple Labarriere (1990) et (2005)
C'est sans doute cette idée de cohérence pratique conduisant à une action globale, cohérence fondée sur la relative autonomie de l'agent, qui incite Aristote à parler du « caractère » (êthos) des animaux dans l'HA, spécialement aux livres VIII et IX. Les animaux ont tous une certaine manière de vivre, conforme aux traits de l'espèce, qui signale en même temps leurs tendances à agir de telle manière plutôt que de telle autre. En cela encore ils sont comme l'homme, dont les vertus éthiques, même si elles ne sont pas « naturelles», traduisent une tendance habituelle à agir ainsi plutôt qu'autrement. Aristote peut donc prêter aux animaux des dispositions que l'on attribuerait volontiers aux êtres humains : le bœuf est doux et dépourvu d'obstination ; le sanglier est plein d'ardeur, obstiné et stupide ; le cerf est prudent et timide, le serpent vile et perfide, etc. ${ }^{28}$ Parmi bien d'autres exemples, mentionnons encore le fait que les chameaux et certains chevaux refusent les saillies incestueuses, de sorte que l'on aurait vu certains d'entre eux réagir violemment pour y avoir été forcés ${ }^{29}$.

Parvenu à ce point de l'analyse, on pourrait se demander si l'éthologie n'a pas rattrapé l'axiologie et la théorie des facultés de l'âme. Si la structure du comportement est identique et si la ressemblance est telle entre l'homme et l'animal qu'elle autorise à parler des constantes comportementales de ce dernier en termes de dispositions morales, on peut alors se poser la question suivante : si l'éthique relève moins des valeurs (le bien, le mal, le juste, l'injuste) que de la conformité à des normes de comportement (le critère de réussite dans l'accomplissement de telle ou telle fonction), alors il n'y a pas d'obstacle majeur à l'attribution d'une certaine éthique à l'animal. Par voie de conséquence, et bien qu'Aristote n'en ait pas lui-même parlé, n'y aurait-il pas sens à élargir la sphère pratique de telle sorte que l'homme agisse « éthiquement » avec l'animal ? Ainsi, en mettant au jour les aspects éthologiques de la zoologie aristotélicienne, certaines études sont allées assez loin dans leur logique pour reconnaître chez Aristote l'existence $d^{\prime}$ une authentique intelligence animale et pour faire des animaux des êtres véritablement « politiques » ${ }^{30}$.

Sans qu'il soit ici possible de reprendre tous les aspects de ce dossier complexe et, par là-même, 
d'arrêter une position définitive, je voudrais au moins prendre l'exemple d'un texte qui, tout en semblant aller dans le sens de ces lectures que l'on dira « continuistes », donne en réalité des arguments servant l'interprétation opposée. Ce texte, HA, VIII ${ }^{31}$, 1, 588a16-b3, est à mon sens décisif étant donné sa position stratégique, au tout début de l'enquête éthologique de l'HA :

Voilà donc comment se présentent la complexion des animaux et leurs modes de reproduction. Quant à leur conduite et à leur genre de vie, ils diffèrent selon leurs mœurs et leur nourriture. Car on trouve chez la plupart des autres animaux eux-mêmes, des traces des états psychologiques qui, chez les hommes, offrent des différences plus marquées. En effet la douceur et la sauvagerie, l'humeur facile et le mauvais caractère, le courage et la lâcheté, les dispositions à la crainte ou à la témérité, les désirs, les fourberies, des ressemblances avec la compréhension intelligente, constituent des ressemblances avec l'homme qu'on retrouve chez beaucoup d'animaux, et qui rappellent les ressemblances dont nous avons parlé à propos des parties du corps. Car certains caractères diffèrent de l'homme selon le plus ou le moins, et il en va de même pour l'homme comparé à un grand nombre d'animaux (en effet, certains de ces états psychologiques atteignent un plus haut degré chez l'homme, certains un degré plus élevé chez les autres animaux), d'autres au contraire diffèrent par analogie : de même qu'il y a chez l'homme art, sagesse, compréhension, de même chez certains animaux il y a quelque autre faculté naturelle du même genre. Cette remarque est particulièrement évidente si l'on considère les enfants dans leur premier âge : chez les enfants, en effet, il est possible de voir comme les traces et les germes de leurs dispositions futures, et l'âme ne diffère pour ainsi dire nullement de l'âme des bêtes durant cette période, si bien qu'il n'est pas illogique que certains caractères soient les mêmes chez l'homme et chez les autres animaux, que d'autres soient très voisins, et que d'autres encore aient des rapports d'analogie $^{32}$. (trad. P. Louis modifiée).

Ce passage a été finement analysé par JeanLouis Labarrière (1990, p. 410-411), qui en a toutefois présenté une lecture continuiste qui me paraît discutable. Il estime en effet que " "l'intelligence relative au raisonnement" < dans ma traduction : « la compréhension intelligente "> fait partie des ressemblances susceptibles de degrés » (LABARRIÈRE, 1990, p. 411), par opposition aux ressemblances simplement fondées sur l'analogie, tout en appartenant également aux ressemblances par analogie. Labarrière semble donc voir un glissement dans le texte ou, selon ses propres termes, « deux niveaux d'analyses ». Il faudrait donc distinguer entre la première et la seconde mention de la sunesis ${ }^{33}$. La première signalerait une différence de degré et la seconde une différence par analogie.

En réalité, il est très peu probable qu'il en aille ainsi, et cela pour au moins trois raisons. (a) Notons d'abord que, dans la première occurrence, Aristote n'attribue pas expressément la sunesis aux animaux, mais seulement - ce qui explique que le

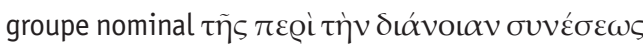
soit au génitif, et non pas au nominatif comme les autres caractères mentionnés - une faculté non nommée qui relève de, qui ressemble, ou qui a trait $\grave{a}$ la sunesis. L'intelligence dont il est ici question est donc, en son sens éminent, une intelligence humaine. L'animal ne fait que lui ressembler par l'une de ses compétences cognitives.

(b) En second lieu, dans la seconde occurrence, Aristote place sans aucune ambiguïté ce qui relève de la sunesis - et donc implicitement cette fameuse « intelligence relative au raisonnement » - sous la catégorie des ressemblances par analogie et non pas sous celle des ressemblances graduelles $^{34}$. Il est assez clair en effet, comme Labarrière semble d'ailleurs l'admettre lui-même, que la proposition « ainsi, à ce qui chez l'homme est art, sagesse, compréhension [...] du même genre » développe le cas de l'analogie et non pas celui de la ressemblance graduelle. C'est ce qu'indiquent, non seulement la place de la phrase et sa conjonction explicative ( $\gamma \dot{\alpha} \varrho)$ avec ce qui la précède - à savoir : « d'autres au contraire diffèrent par analogie »-, mais encore et surtout sa syntaxe comparative en

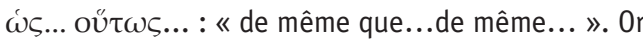
Aristote oppose nettement les deux cas : les caractères relèvent soit de la différence graduelle, soit de la différence par analogie. La structure adversative
31. Ou livre VII selon l'ordre des manuscrits, adopté par Balme (1991).

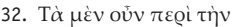

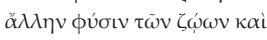

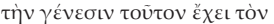

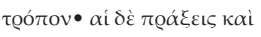

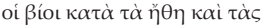

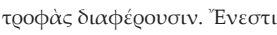

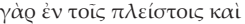
$\tau \tilde{\omega} \nu \alpha \alpha \lambda \lambda \omega \nu \zeta \omega \omega v$ í $\chi v \eta \tau \bar{\omega} v$

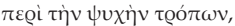

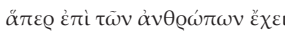

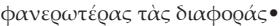

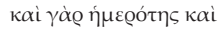

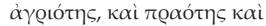
$\chi \alpha \lambda \varepsilon \pi$ ót门

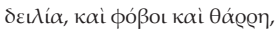

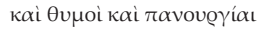

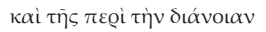

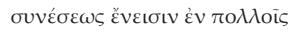

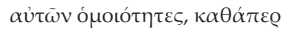

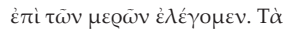
$\mu \dot{\varepsilon} v \gamma \dot{\alpha} \varrho \tau \tilde{\omega} \mu \tilde{\alpha} \lambda \lambda \mathrm{ov} \kappa \alpha \grave{i} \hat{\eta} \tau \tau o v$

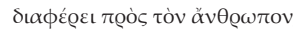

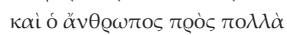
$\tau \tilde{\omega} \nu \zeta \omega \omega \nu$ (

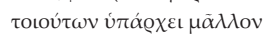

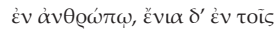

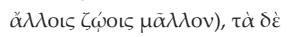

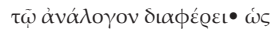

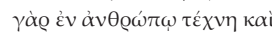

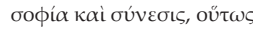

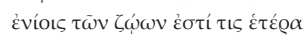

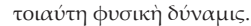

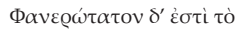

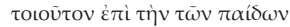

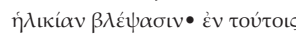

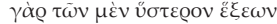

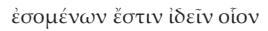

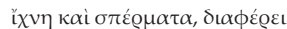

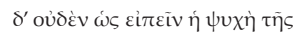

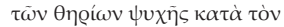

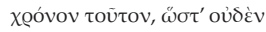

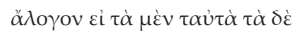

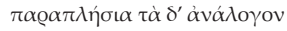
v́tá 33. Voir en ce sens: LABARRIERE, 1990, p. $410 ; 414$.

34. Voir : BALME, 1991, p. 59, n.b, d. 


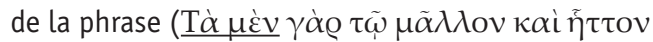

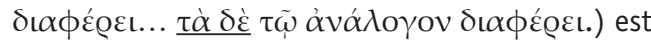
très claire. Aristote n'envisage aucunement un genre mixte et, de fait, on ne voit pas comment ce qui ressemble par analogie pourrait également différer par degrés. Lorsqu'il arrive à Aristote de parler d'intelligence animale et d'employer pour ce faire un vocabulaire en principe réservé à l'intelligence humaine, il faut donc convenir qu'il le fait pour désigner un autre type de compétence, et non pas une même compétence qui, chez l'animal, serait simplement moins développée ${ }^{35}$.

La situation est donc la suivante : il y a de Balme à propos de $\mathrm{HA}$ VIII, 1, 588a23-24 : « Arist. does not credit animals with human intelligence either here or elsewhere, but only with resemblances and analogies (BALME, 1991, p. 59, n.b) 36. La connaissance des ressemblances contribue à définition, notamment quand elle fait apparaître que les cas similaires appartiennent à un même genre : Topiques, I, 18 $108 \mathrm{~b} 19-31$

37. $H A, I, 1,488 \mathrm{~b} 24-26$ 38. BALME, 1991, p. 58

3. $E E$, II, 6, 1233a4-10 animaux en termes anthropomorphiques et moraux, conformément à une représentation populaire du comportement animal $^{38}$, avant d'évoquer l'intelligence humaine : ce faisant, il indique implicitement que cette attribution n'implique pas qu'il faille attribuer à l'animal le type de délibération - morale ou non - dont l'homme est capable.

Pour dire les choses avec un peu plus de distance, le point de vue éthologique ne menace pas la différence anthropologique et ne risque pas de « naturaliser » l'éthique aristotélicienne : il montre au contraire avec finesse ce qu'il y a de commun entre action humaine et action animale pour mettre en évidence très précisément ce en quoi elles diffèrent.

\section{Conclusion}

L'autonomie animale et la décision humaine ne sauraient former qu'un couple en miroir. L'homme semble bel et bien posséder une faculté spécifique par rapport à celle de l'animal, ce qui le conduit à mener une vie proprement morale et à agir de manière délibérée et non pas selon les lois et les circonstances naturelles qui définissent le comportement. Capable de délibérer et de choisir, il peut prendre un parti ou son contraire ${ }^{39}$, obéir à la raison droite ou s'y soustraire par une opinion viciée, ce qui suppose qu'il ne soit pas intégralement déterminé par la causalité naturelle. Au-delà des analogies incontestables que dessine l'analyse éthologique, et du fait même qu'il ne s'agit que d'analogies, la discontinuité l'emporte lorsqu'il s'agit de distinguer entre action animale et action humaine. Ainsi, prendre en compte l'animal dans l'analyse de l'action aide sans nul doute à la compréhension théorique de celle-ci, mais cela ne saurait contribuer à sa véritable mise en œuvre, c'est-à-dire à la pratique.

Plus fondamentalement, ce qui caractérise l'homme parmi les animaux, ce n'est pas de posséder un degré de détermination supérieur, mais au contraire d'être moins déterminé. Il possède certes une faculté que les animaux ne possèdent pas, sinon par une sorte d'homonymie, ce qui est en soi une détermination supplémentaire, mais cette faculté introduit une indétermination constitutive : elle qu'Aristote attribue des traits de caractères aux 
renvoie l'homme à ses propres virtualités et fonde ainsi la possibilité d'une morale. Délibérer, cela suppose que l'on puisse toujours agir ainsi plutôt qu'autrement, et non pas seulement accomplir une fonction définie par la nature. Ce qui différencie d'abord l'homme de l'animal, c'est donc une différence de puissances. Celles de l'homme le conduisent à se trouver condamné à choisir, et à pouvoir le faire entre deux partis contraires, de sorte que sa praxis, à la différence de la praxis animale, n'est jamais simplement un phénomène mais toujours aussi un événement.

On pourrait croire que l'éthologie fait obstacle à la différence anthropologique, ou vouloir qu'elle le fasse. Les traités zoologiques, en réalité, montrent plutôt l'inverse : en posant les ressemblances entre l'action humaine et l'action animale, ils définissent le genre commun à l'intérieur duquel celles-ci peuvent être distinguées. Paradoxe fécond, qui invite à se défaire des clivages artificiels et des lectures forcées : celle qui oppose irréductiblement la pratique humaine au comportement animal, l'éthique à l'éthologie, comme celle qui voudrait à toute force naturaliser l'agir humain.

\section{BIBLIOGRAPHIE}

BALME, D. (1991). Aristotle. History of Animals, Books VII-X. Edited and Translated. Harvard University Press, Cambridge-London.

BRUNSCHWIG, J. (1986). The Cradle Argument in Epicureanism and Stoicism. In: SCHOFIELD, M. ; STRIKER, G. (Eds). The Norms of Nature. Studies in Hellenistic Ethics, Cambridge - Paris, 1986, p. 113-144.

LABARRIÈRE, J.-L. (1990). De la phronesis animale. In : DEVEREUX, D. ; PELLEGRIN, P. (Dir.). Biologie, logique et métaphysique chez Aristote, Actes du Séminaire C.N.R.S. N.S.F., Oléron, 28 juin-3 juillet 1987, Paris, 1990, p. 405428

(1993). Aristote et l'éthologie, Revue Philosophique de la France et de l'Étranger, 1993/2, p. 281-300.

(2004). Langage, vie politique et mouvement des animaux. Paris, Vrin, 2004.

(2005). La Condition animale. Études sur Aristote et les stoïciens. Louvain-La-Neuve, Éditions Peeters, 2005.

MOREL, P.-M. (2007). De la matière à l'action. Aristote et le problème du vivant. Paris, Vrin, 2007.

. (2012). Genèse, analogie, dépassement. Les voies du naturalisme aristotélicien. In : HABER, S. ; MACÉ, A. (éds). Anciens et Modernes par-delà nature et société. Presses universitaires de Franche-Comté, Besançon, 2012, p. 85-101.

. (2013). Aristote, Le Mouvement des animaux la Locomotion des animaux. Paris, GF-Flammarion, 2013.

PAKALUK, M. (2011). Mixed Actions and Double Effect. In: PAKALUK, M. ; PEARSON, G. (Eds). Moral Psychology and Human Action in Aristotle. 0xford, New-York, Oxford University Press, 2011, p. 211-231.

SORABJI, R. (1993). Animal Minds and Human Morals. The Origins of the Western Debate. London, Duckworth, 1993.

Recebido em setembro de 2012 e aprovado em setembro de 2012. 